\title{
Etude expérimentale du soudage par laser YAG de l'alliage base nickel Hastelloy $X$
}

\section{Experimental study of YAG laser welding of nickel base alloy Hastelloy $X$}

\author{
Jérémie Graneix ${ }^{1}$, Jean-Denis Beguin ${ }^{1}$, François Pardeilhan ${ }^{2}$, Talal Masri ${ }^{1}$ et Joël Alexis ${ }^{1}$ \\ ${ }^{1}$ ENIT, Ecole Nationale d'Ingénieurs de Tarbes, France \\ ${ }^{2}$ EXAMECA, France
}

\begin{abstract}
The YAG laser welding process is contemplated to replace the manual TIG welding process for the production of parts of turbojet in Hastelloy X. This experimental study has identified the field of weldability of this alloy to meet the specific requirements of the aerospace industry.

Résumé. Le procédé de soudage laser YAG est envisagé pour remplacer le procédé de soudage TIG manuel pour la réalisation de pièces de turboréacteur en alliage nickel-chrome-molybdène Hastelloy X. Cette étude expérimentale a permis de définir un domaine de soudabilité de cet alliage répondant aux critères spécifiques du secteur aéronautique.
\end{abstract}

\section{INTRODUCTION}

L'alliage à durcissement par solution solide Hastelloy X possède une résistance à l'oxydation, une résistance à haute température ainsi qu'une formabilité importantes ; critères sine qua none pour le choix des matériaux pour la réalisation de chambres de combustion de turboréacteur qui constitue le cadre de cette étude [1-3].

Les exigences des pièces aéronautiques mécanosoudées devenant de plus en plus sévères notamment en termes de reproductibilité de géométrie et de qualité métallurgique des cordons, le soudage laser devient une méthode d'assemblage envisageable pour respecter ces nouvelles contraintes de par son automatisation permettant de remplacer à plus long terme le soudage manuel par procédé TIG. L'objet de cette étude est donc de déterminer le domaine de soudabilité de l'alliage Hastelloy X par le procédé de soudage laser YAG.

\section{MATÉRIAU ET PROCÉDURES EXPÉRIMENTALES}

L'alliage étudié au cours de cette étude est l'alliage nickelchrome-molybdène Hastelloy X (NiCr22Fe18Mo9Co2W selon UNS : N06002) dont la composition est donnée dans le tableau 1. La tôle d'épaisseur 1,2 $\mathrm{mm}$ a donc été mise en solution entre 1150 et $1175^{\circ} \mathrm{C}$ et refroidit rapidement. Le traitement et le refroidissement sont réalisés sous atmosphère protectrice. Les coupons de matière ont été soudés en configuration bord à bord sous protection gazeuse d'azote avec une machine TruLaser Cell 3000 de chez TRUMPH.

La source laser est une source Nd : YAG TruDisk de puissance maximale $3,3 \mathrm{~kW}$. Afin d'identifier les paramètres influents du procédé de soudage tout en rationalisant les essais, un plan d'expérience a été établi à l'aide du logiciel CORICO. Quatre paramètres ont été retenus pour cette étude préliminaire : la puissance laser, la vitesse d'avance, le diamètre focal et le débit de gaz.
Tableau 1. Composition of the alloy nickel base NiCr22Fe18Mo9Co2W sheet (wt. \%).

\begin{tabular}{lllllllll}
\hline $\mathrm{Ni}$ & $\mathrm{Cr}$ & $\mathrm{Co}$ & $\mathrm{Mo}$ & $\mathrm{W}$ & $\mathrm{Fe}$ & $\mathrm{Mn}$ & $\mathrm{Si}$ & $\mathrm{C}$ \\
\hline Bal & 22,1 & 1,38 & 9,0 & 0,45 & 18,7 & 0,66 & 0,35 & 0,07 \\
\hline
\end{tabular}

La position du point focal par rapport à la surface de la tôle n'a pas été étudiée car de nombreuses recherches dans ce domaine ont montré une pénétration maximum à une distance focale de $-1 / 3$ de l'épaisseur de la tôle par rapport à la surface et ceci quelques soient le diamètre focal ou la vitesse d'avance. Cette étude s'est déroulée en deux étapes : une étude préliminaire a été réalisée avec le logiciel de plan d'expérience CORICO en balayant les 4 paramètres choisis de la manière la plus large possible afin de réduire le domaine de soudabilité défini à partir de critères géométriques des cordons. La deuxième étude a permis de déterminer un domaine de soudabilité plus restreint mettant en évidence l'influence de chaque paramètre sur la qualité du joint soudé.

\section{RÉSULTATS EXPÉRIMENTAUX}

\section{Matière de base}

La microstructure de la tôle a été étudiée à partir d'observations par microscopie optique et par microscopie électronique à balayage (MEB-FEG JEOL 7000F) couplées à des analyses EBSD (caméra OXFORD Nordlys Fast). La microstructure présente des grains équiaxes de taille de l'ordre de $10 \mu \mathrm{m}$ quel que soit le plan de la tôle étudiée. La structure CFC correspond à celle de la solution solide riche en nickel. Les cartographies IPF Z0 des plans L-TC et TC-TL montrent que la tôle ne présente pas de texture marquée (Fig. 1).

Les propriétés mécaniques en traction suivant différentes directions dans le plan L-TL ont été 

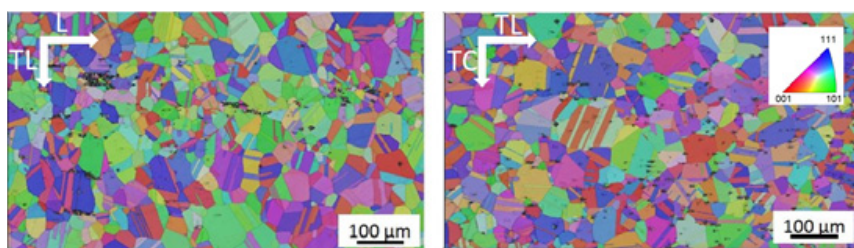

Figure 1. Surface normal-projected inverse pole figure orientation map (IPF Z0) of the alloy nickel base $\mathrm{NiCr} 22 \mathrm{Fe} 18 \mathrm{Mo} 9 \mathrm{Co} 2 \mathrm{~W}$ sheet.

Tableau 2. Mechanical properties of the alloy nickel base $\mathrm{NiCr} 22 \mathrm{Fe} 18 \mathrm{Mo} 9 \mathrm{Co} 2 \mathrm{~W}$ sheet.

\begin{tabular}{|c|c|c|c|c|c|}
\hline & \multicolumn{2}{|c|}{ Essais de traction } & \multicolumn{3}{|r|}{ Dureté } \\
\hline & $\mathrm{E}(\mathrm{GPa})$ & $\operatorname{Re}(\mathrm{MPa})$ & $\mathrm{Rm}(\mathrm{MPa})$ & $\mathrm{A}(\%)$ & $\mathrm{HV}_{5}$ \\
\hline$\overline{0^{\circ}}$ & $208 \pm 10$ & $445 \pm 5$ & $799 \pm 2$ & $67 \pm 10$ & $\overline{22 \pm 6}$ \\
\hline $45^{\circ}$ & $211 \pm 15$ & $442 \pm 10$ & $793 \pm 11$ & $63 \pm 2$ & \\
\hline $90^{\circ}$ & $190 \pm 20$ & $455 \pm 5$ & $806 \pm 5$ & $60 \pm 3$ & \\
\hline
\end{tabular}
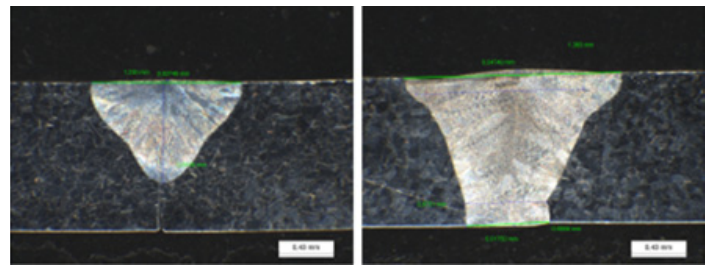

Figure 2. Metallographic observations of welds obtained with the welding parameters (power, translation speed, focal diameter and gas flow) (a) $1650 \mathrm{~W}, 6,25 \mathrm{~m} / \mathrm{min}, 500 \mu \mathrm{m}, 10 \mathrm{l} / \mathrm{min}$, (b) $2500 \mathrm{~W}$, $8 \mathrm{~m} / \mathrm{min}, 650 \mu \mathrm{m}, 40 \mathrm{l} / \mathrm{min}$.

déterminées ainsi que la dureté Vickers (Tableau 2). Aucune anisotropie n'est mise en évidence confirmant les observations microstructurales précédentes.

\section{Joints soudés}

Afin de valider la soudabilité de cet alliage, défini à partir de critères géométriques des cordons décrits dans la norme EN ISO 6947, des coupes métallographiques des joints dans le plan TL-TC ont été réalisées (Fig. 2).

Ces différentes observations ont permis de déterminer le domaine de soudabilité en fonction de la vitesse de translation, de la puissance de la source et du diamètre focal (Fig. 3).

La représentation iconographique donnée par le logiciel de plan d'expérience CORICO (Fig. 4) permet de montrer que la profondeur de pénétration augmente si le diamètre focal ou si la vitesse de translation diminuent. De plus, la largeur du cordon augmente avec la puissance ou bien encore le débit de gaz.

Les propriétés mécaniques des cordons ont été déterminées à l'aide d'essais de traction réalisés dans la direction TL sur des éprouvettes prélevées dans le plan L-TL. Les résultats de ces essais, obtenus pour des éprouvettes répondant aux critères géométriques précédemment étudiés, sont présentés à la figure 5 . Quatre éprouvettes, soudées différemment, présentent des propriétés mécaniques identiques et maximales.

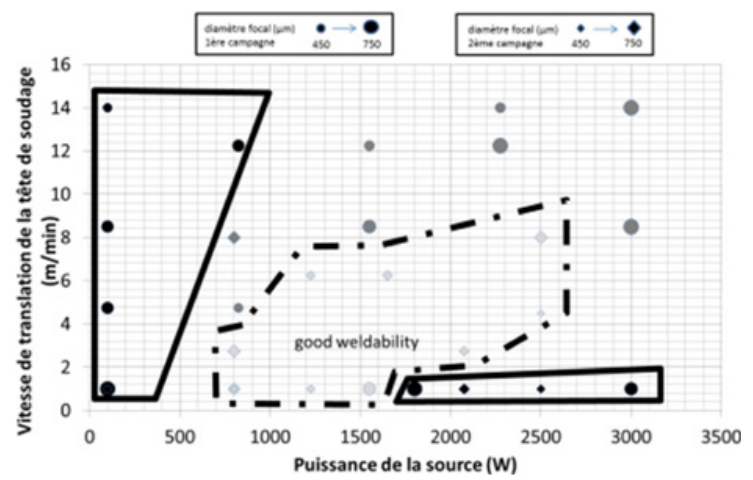

Figure 3. Welding penetration function to the welding parameters.

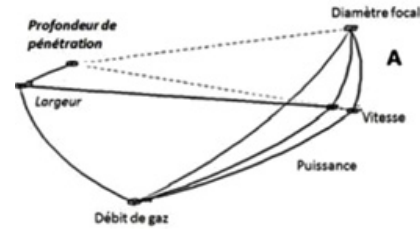

Figure 4. Graphic iconographic relations given by the software CORICO.

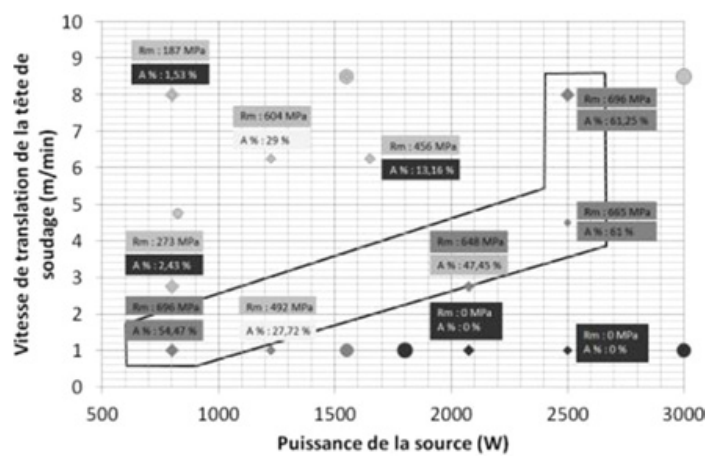

Figure 5. Ultimate tensile stress and elongation to failure of samples welded function to the welding parameters.

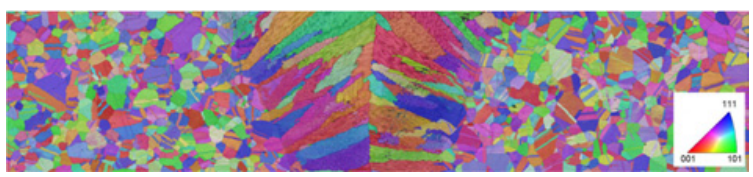

Figure 6. Surface normal-projected inverse pole figure orientation map (IPF Z0) of the weld $(2500 \mathrm{~W}, 8 \mathrm{~m} / \mathrm{min}, 650 \mu \mathrm{m}$, 401/min).

La microstructure du joint soudé à $2500 \mathrm{~W}$ avec une vitesse de translation de $8 \mathrm{~m} / \mathrm{min}$ a été caractérisée par EBSD (Fig. 6). La zone fondue présente de larges grains dendritiques en comparaison aux grains équiaxes du matériau de base. La zone affectée thermique n'est pas perceptible.

\section{CONCLUSIONS}

La mise en œuvre du logiciel de plan d'expérience CORICO a permis d'obtenir en quelques essais le domaine de soudabilité de l'alliage $\mathrm{NiCr} 22 \mathrm{Fe} 18 \mathrm{Mo} 9 \mathrm{Co} 2 \mathrm{~W}$ par soudage laser YAG en configuration bord à bord. Les 
paramètres d'ordre un de soudage ont été déterminés. Les transformations métallurgiques lors du soudage ainsi que la durabilité des cordons doivent cependant être approfondies.

\section{Références}

[1] Hastelloy $\mathrm{X}$ alloy, Haynes international report H-3009C, 1997, 1-16.
[2] Fabrication of Haynes and Hastelloy solidsulution-strenghtened high-temperature alloys, Haynes international report H-3159CA, 2002, $1-58$.

[3] C. R. Brinkman, P. L. Rittenhouse, W. R. Corwin, J. P. Strizak, and A. Lystrup J. R. Distefano. Application of Hastelloy $X$ in gaz cooled reactor Systems. OAK Ridge National Laboratory, 1976. 